ARTICLE

\title{
Probing QED and fundamental constants through laser spectroscopy of vibrational transitions in $\mathrm{HD}^{+}$
}

J. Biesheuvel', J.-Ph. Karr²,3, L. Hilico ${ }^{2,3}$, K.S.E. Eikema', W. Ubachs ${ }^{1}$ \& J.C.J. Koelemeij ${ }^{1}$

The simplest molecules in nature, molecular hydrogen ions in the form of $\mathrm{H}_{2}^{+}$and $\mathrm{HD}^{+}$, provide an important benchmark system for tests of quantum electrodynamics in complex forms of matter. Here, we report on such a test based on a frequency measurement of a vibrational overtone transition in $\mathrm{HD}^{+}$by laser spectroscopy. We find that the theoretical and experimental frequencies are equal to within 0.6(1.1) parts per billion, which represents the most stringent test of molecular theory so far. Our measurement not only confirms the validity of high-order quantum electrodynamics in molecules, but also enables the long predicted determination of the proton-to-electron mass ratio from a molecular system, as well as improved constraints on hypothetical fifth forces and compactified higher dimensions at the molecular scale. With the perspective of comparisons between theory and experiment at the 0.01 part-per-billion level, our work demonstrates the potential of molecular hydrogen ions as a probe of fundamental physical constants and laws.

\footnotetext{
${ }^{1}$ LaserLaB, Department of Physics and Astronomy, VU University, De Boelelaan 1081, Amsterdam $1081 \mathrm{HV}$, The Netherlands. ${ }^{2}$ Laboratoire Kastler Brossel, UPMC-Sorbonne Universités, CNRS, ENS-PSL Research University, Collège de France, 4 place Jussieu, Paris 75005, France. ${ }^{3}$ Département de Physique, Université d'Evry Val d'Essonne, Boulevard François Mitterrand, 91025 Evry, France. Correspondence and requests for materials should be addressed to J.C.J.K. (email: j.c.j.koelemeij@vu.nl).
} 
T he possibility of accurate quantum electrodynamics (QED) calculations and the presence of narrow optical transitions between long-lived vibrational levels make laser spectroscopy of $\mathrm{H}_{2}^{+}$and $\mathrm{HD}^{+}$a sensitive tool to test fundamental physics at the molecular scale. Examples include tests of relativistic quantum mechanics and $\mathrm{QED}^{1-4}$, and searches for physics beyond the Standard Model at the molecular scale ${ }^{5,6}$ and beyond General Relativity ${ }^{7,8}$. Furthermore, spectroscopy of the molecular hydrogen ion has long been proposed as a means to determine the value of fundamental physical constants ${ }^{1,2,4,9}$, and to realise precise molecular clocks 8 .10.

Nearly all the above applications involve a comparison of stateof-the-art molecular theory with accurate results from (most often laser) spectroscopy. The most accurate results so far have been obtained using $\mathrm{HD}^{+}$ions stored in radiofrequency (r.f.) traps, sympathetically cooled by laser-cooled $\mathrm{Be}^{+}$ions ${ }^{3,11}$, allowing tests at a relative uncertainty of a few parts per billion (p.p.b.). However, the most precise experimental result ${ }^{3}$, a frequency measurement of the $(v, L):(0,0)-(1,1)$ rovibrational line at $5.1 \mu \mathrm{m}$ in $\mathrm{HD}^{+}$with a relative uncertainty of 1.1 p.p.b., was found to disagree by 2.7 p.p.b. with a more accurate theoretical value obtained from state-of-the-art ab initio molecular theory ${ }^{1,2}$. This disagreement has thus far been unresolved, and additional high-precision experimental data are needed to draw conclusions about the validity of the theoretical framework, and to open up the wide range of applications of molecular hydrogen ion spectroscopy mentioned above.

Here, we report on an optical frequency measurement of the $(v, L):(0,2)-(8,3)$ vibrational overtone transition in $\mathrm{HD}^{+}$at $782 \mathrm{~nm}$. Our experimental result is found to be in agreement with the theoretical prediction to within 0.6(1.1) p.p.b., thereby confirming the validity of relativistic quantum mechanics and QED in a vibrating molecular system at an unprecedented level. We subsequently exploit the agreement between theory and experiment for the first determination of the proton-electron mass ratio from a molecular system, and to put tighter constraints on the strength and range of 'fifth forces' at the molecular scale.

\section{Results}

Experimental procedure. Our experimental apparatus and procedure for $\mathrm{HD}^{+}$spectroscopy are described in detail in the Methods section. In brief, trapped $\mathrm{HD}^{+}$molecular ions are cooled to $\sim 10 \mathrm{mK}$ by storing them together with laser-cooled $\mathrm{Be}^{+}$ions, and we excite and detect the $(v, L):(0,2)-(8,3)$ transition by resonance-enhanced $\left(1+1^{\prime}\right)$ multiphoton dissociation (REMPD) spectroscopy, see Fig. 1a. We acquire a spectrum by observing the loss of $\mathrm{HD}^{+}$because of REMPD, inferred from the change in the monitored $\mathrm{Be}^{+}$fluorescence induced by r.f. excitation of the $\mathrm{HD}^{+}$secular motion, for different values of the $782 \mathrm{~nm}$ spectroscopy laser frequency (Methods and Fig. 2a). The latter is stabilized and counted using an optical frequency comb laser (Methods).

Line shape model for nonlinear least-squares fitting. Hyperfine interactions lead to a manifold of 83 lines (Fig. 1b,c), the $\sim 25$ strongest of which are located within the range $(-110 \mathrm{MHz}, 140 \mathrm{MHz})$ around the hyperfine-less rovibrational frequency, $v_{0}$. Each hyperfine component is Doppler broadened to $\sim 16 \mathrm{MHz}$, and the hyperfine structure is only partially resolved. Using a realistic spectral line shape function (Methods), we employ nonlinear least squares fitting to extract not only the transition frequency $v_{0, \text { fit }}$, but also the intensity of the $782 \mathrm{~nm}$ laser, $I_{\mathrm{L}}$, the motional temperature of the $\mathrm{HD}^{+}$ions, $T_{\mathrm{HD}+}$, and the temperature of the $\mathrm{Be}^{+}$ions during secular excitation, $T_{\mathrm{i}}$, which are relevant parameters for the spectral analysis (see Methods for details). The 782-nm intensity we find agrees (within the fit error) with the intensity estimated from the laser beam waist and beam alignment uncertainty. Likewise, the fitted temperature agrees well with the results of molecular dynamics (MD) simulations.

The relevance of the additional fit parameters is illustrated by the correlation matrix, $\Omega$, of the estimated parameters, which reveals significant correlations between $v_{0, \text { fit }}$ and $T_{\mathrm{HD}+}$, and $v_{0, \text { fit }}$ and $I_{\mathrm{L}}$ (Fig. 2c). Adding fit parameters results in an increased error in the fitted value $v_{0, \text { fit }}$, which rises from $0.25 \mathrm{MHz}$ (0.65 p.p.b. relative to $v_{0, \text { fit }}$ ) to $0.33 \mathrm{MHz}\left(0.85\right.$ p.p.b.) when $T_{\mathrm{i}}$, $T_{\mathrm{HD}+}$ and $I_{\mathrm{L}}$ are added as free fit parameters. Even so, the spectral fit result presented here marks the first time that a sub-p.p.b. resolution is achieved in molecular-ion spectroscopy.

Systematic effects and frequency value. The fit result $v_{0, \text { fit }}$ is corrected for various systematic frequency offsets because of electric and magnetic fields. We calculate the a.c. and d.c. Stark effect $a b$ initio with high accuracy following the approach of Karr ${ }^{10}$ to find the frequency shift because of blackbody radiation (BBR), the r.f. trap field and the electric fields of the lasers. The total Stark shift of $-1.3(1) \mathrm{kHz}$ is dominated by the shifts due to the 313, 532 and $782 \mathrm{~nm}$ lasers (which are all on during excitation), with individual contributions of $0.008(1) \mathrm{kHz}$, $-0.45(7) \mathrm{kHz}$ and $-0.87(13) \mathrm{kHz}$, respectively. The uncertainty of the Stark shift is caused almost entirely by the uncertainties of the laser beam intensities. We also evaluate the Zeeman effect, which stems from the static quantization field of $0.19 \mathrm{mT}$ directed along the $z$ axis of the trap (Methods). The polarization of the $782 \mathrm{~nm}$ laser is such that it induces $\sigma^{-}$and $\sigma^{+}$transitions at equal rates. The Zeeman effect leads to a shift to $v_{0, \text { fit }}$ by $-16.9(3.2) \mathrm{kHz}$, the uncertainty of which is due to a possible $2 \%$ imbalance between the $\sigma^{-}$and $\sigma^{+}$rates caused by imperfect polarization optics and depolarization because of the windows of the vacuum chamber. Another uncertainty stems from the accuracy of the theoretical hyperfine structure ${ }^{12}$, which enters through our spectral line shape function. We repeated the fit procedure with a spectral line shape function based on the hyperfine structure obtained with slightly altered spin coefficients (within their uncertainty range), for which we observe essentially no shift of $v_{0, f i t}$. Compared with the $0.33 \mathrm{MHz}$ statistical fit uncertainty of $v_{0, \text { fit }}$, the contribution of the above line shifts to the total frequency value and uncertainty is negligible, as are the frequency shifts due to the second-order Doppler effect ${ }^{13}$ $(<5 \mathrm{~Hz})$ and the electric-quadrupole $\operatorname{shift}^{14}(<0.1 \mathrm{kHz})$.

During REMPD, the motional dynamics in the laser-cooled Coulomb crystal are significantly influenced by several (laser-induced) chemical processes. For example, REMPD of an $\mathrm{HD}^{+}$ion leads to the formation of energetic fragments, and previously it was observed that the two reactions, $\mathrm{HD}^{+}+h v_{1}+$ $h v_{2} \rightarrow \mathrm{H}(1 \mathrm{~s})+\mathrm{D}^{+}+$k.e. and $\mathrm{HD}^{+}+h v_{1}+h v_{2} \rightarrow \mathrm{D}(1 \mathrm{~s})+\mathrm{H}^{+}$ + k.e., occur with about equal probability (here k.e. stands for kinetic energy, and $v_{1}$ and $v_{2}$ for the frequencies of the 782 and $532 \mathrm{~nm}$ lasers, respectively) ${ }^{15}$. Owing to their relatively high charge-to-mass ratio, the produced protons are not stably trapped in our apparatus. Indeed, we observe only cold, trapped deuterons after REMPD, indicating that each deuteron transferred most of its $0.41 \mathrm{eV}$ kinetic energy to the ions in the Coulomb crystal (which itself contains only $2 \mathrm{meV}$ of thermal energy at $10 \mathrm{mK}$ ), causing (at least transiently) significant heating of the crystal. Other reactions with energetic ionic products are the result of collisions with $\mathrm{H}_{2}$ molecules in the background vapour, and we also take the reactions $\mathrm{Be}^{+}\left({ }^{2} \mathrm{P}_{3 / 2}\right)+\mathrm{H}_{2} \rightarrow$ $\mathrm{BeH}^{+}+\mathrm{H}(1 \mathrm{~s})+$ k.e., $\mathrm{HD}^{+}+\mathrm{H}_{2} \rightarrow \mathrm{H}_{2} \mathrm{D}^{+}+\mathrm{H}(1 \mathrm{~s})+$ k.e. and $\mathrm{HD}^{+}+\mathrm{H}_{2} \rightarrow \mathrm{H}_{3}^{+}+\mathrm{D}(1 \mathrm{~s})+$ k.e. (with ionic product kinetic 
a

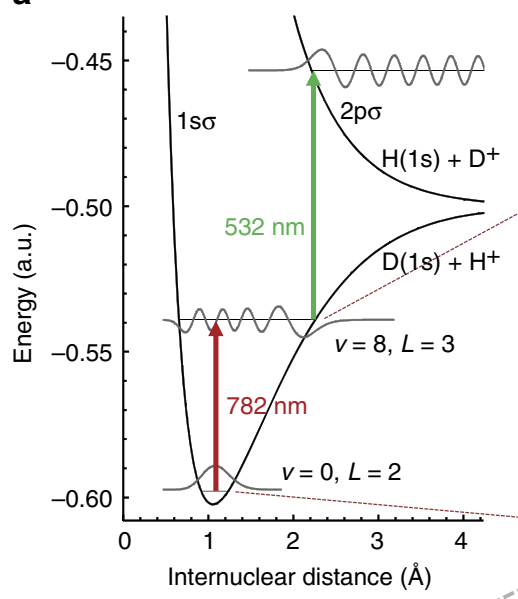

b




Figure 1 | Partial level diagram, excitation scheme and interaction with blackbody radiation. The $(v, L):(0,2)-(8,3)$ rovibrational transition is excited and detected through REMPD by 782 and $532 \mathrm{~nm}$ laser radiation (a, solid arrows), which leads to the loss of $\mathrm{HD}^{+}$by dissociation into either the $\mathrm{H}(1 \mathrm{~s})+\mathrm{D}^{+}$or the $\mathrm{D}(1 \mathrm{~s})+\mathrm{H}^{+}$channel ${ }^{15}$. Magnetic interactions between the proton spin $\mathbf{I}_{\mathrm{p}}$, deuteron spin, $\mathbf{I}_{\mathrm{d}}$, electron spin, $\mathbf{s}_{\mathrm{e}}$, and molecular rotation, $\mathbf{L}$, lead to hyperfine structure in the excitation spectrum $(\mathbf{b}, \mathbf{c})$. Blackbody radiation induces rotational transitions (b, dashed arrows) between $v=0, L=2$ and states with $L=1,3$ which are in turn coupled to $L=0,4$ (not shown), and the population in each hyperfine state depends on the balance between the REMPD rate and the rate at which BBR replenishes population. These effects are included in a hyperfine rate equation model, which is used to obtain a realistic spectral line shape function. Treating this at the level of individual hyperfine states naturally subdivides the $\mathrm{HD}^{+}$population into different spin configuration classes, which are only weakly coupled by the electric dipole transitions induced by the lasers and BBR, and therefore evolve independently during REMPD. (d) Rotational distribution of $\mathrm{HD}^{+}(v=0)$ at an ambient BBR temperature of $300 \mathrm{~K}$. The population of the $v=0, L=2$ initial state amounts to $27 \%$.

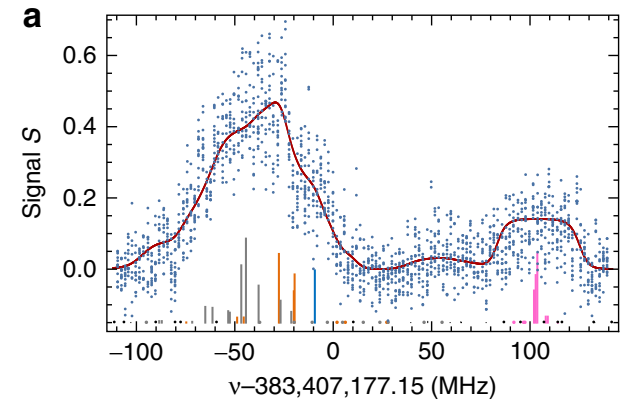

b

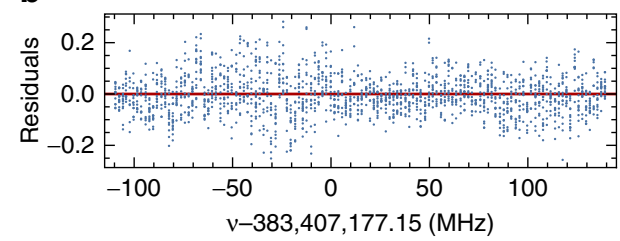

C


Figure 2 | Spectral line shape function and fit to REMPD spectrum. (a) Measured dimensionless REMPD signal $\mathrm{S}$ versus $782 \mathrm{~nm}$ laser frequency (blue dots) and fitted line shape function (red curve). The underlying individual hyperfine components are also shown, following the same colour coding as used in Fig. 1b. The hyperfine components are offset vertically for clarity. (b) Fit residuals versus $782 \mathrm{~nm}$ laser frequency (blue dots). (c) The correlation matrix, $\Omega$, reveals correlations between $v_{0, \text { fit }}$ and the other fitted parameters, and the uncertainty in the fitted value $v_{0, \text { fit }}$ rises from $0.25 \mathrm{MHz}(0.65$ p.p.b. relative to $v_{0, \text { fit }}$ ) to $0.33 \mathrm{MHz}$ (0.85 p.p.b.) when $T_{\mathrm{i}}, T_{\mathrm{HD}}+$ and $I_{\mathrm{L}}$ are added as free fit parameters. (d) Dimensionless spectral line shape function $\mathrm{G}$ obtained with the rate equation model (Methods) assuming $I_{L}=1 \times 10^{7} \mathrm{Wm}^{-2}$ and $T_{\mathrm{Be}+\text {, av }}=4 \mathrm{~K}$, showing the non-trivial combined effect of Doppler broadening, line overlap and saturation versus $\mathrm{HD}^{+}$temperature (axis shown in perspective).

energies of $0.25,0.36$ and $0.66 \mathrm{eV}$, respectively) into account here. We determine reaction rates from measured loss rates of $\mathrm{Be}^{+}$ and $\mathrm{HD}^{+}$, which are in agreement with expected Langevin reaction rates given the pressure of $1 \times 10^{-8} \mathrm{~Pa}$ in our vacuum apparatus ${ }^{16}$. We include these processes in realistic MD simulations (Methods) employing leapfrog integration with an 
adaptive time step, to ensure that collisions between energetic ions are correctly handled. Under these conditions, our MD simulations reveal average $\mathrm{HD}^{+} z$-velocity distributions, which deviate significantly from thermal (Gaussian) distributions, an effect that has hitherto been neglected by the widespread assumption that laser-cooled ion ensembles exhibit thermal velocity distributions. As shown in Fig. 3, we empirically find that the velocity distributions are better represented by $q$-Gaussians ${ }^{17}$, which have the additional advantage that the effect of a given chemical reaction can be parameterized by a corresponding $q$-value (with $q=1$ corresponding to a Gaussian distribution). Taking all reactions and reaction rates into account, we find that the velocity distribution is best characterized by a $q$-Gaussian with $q$ ranging between 1.00 and 1.07, depending on the assumed thermalization rate and the REMPD rate. We therefore include $q$-Gaussians in our spectral line shape function (Methods). Comparing scenarios with minimum and maximum expected thermalization rates (corresponding to $q=1.07$ and $q=1.00$, respectively), we find a mean shift of $-0.25(25) \mathrm{MHz}$ with respect to the case of $q=1.00$. The origin of this shift lies in the overlap and saturation of Doppler-broadened hyperfine components in the spectrum (Fig. 2d). A similar frequency shift may occur when micromotion sidebands are present. Here we make a distinction between excess radial micromotion caused by radial static offset fields, and axial micromotion that could arise

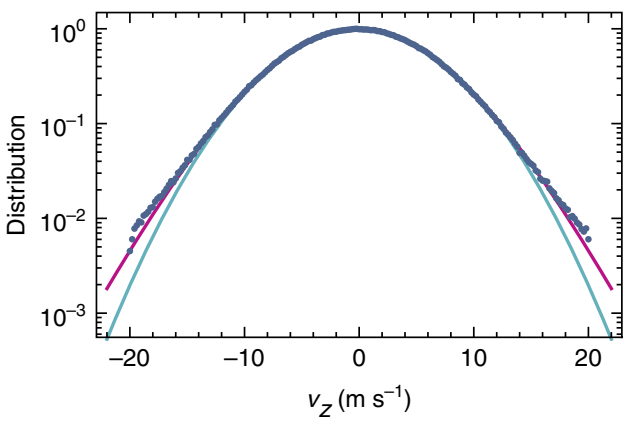

Figure 3 | Non-thermal velocity distributions. When kinematic effects of (laser-induced) chemistry are included in MD simulations, the $\mathrm{HD}^{+}$ $z$-velocity distribution exhibits non-thermal distributions with pronounced tails (blue data points). Indeed, a least-squares fit with a $q$-Gaussian (magenta curve, with fitted temperature $T=11.00$ (3) $\mathrm{mK}$ and $q=1.070$ (3) produces a visibly better result than a fit with a thermal (normal) distribution (turquoise curve, fit with $T=11.60$ (3) $\mathrm{mK}$ ). from an axial r.f. field because of geometrical imperfections of our ion trap. A finite-element analysis of the trap's electric field reveals that such an axial field will be approximately constant over the extent of the Coulomb crystal, which implies that the corresponding micromotion contribution to the line shape will be homogeneous. We use the fluorescence correlation method of Berkeland et al. ${ }^{13}$ to find a small micromotion amplitude of 11(4) $\mathrm{nm}$ amplitude along the 782-nm laser beam as follows. Measurements of the r.f. field amplitude are performed on the $\mathrm{Be}^{+}$ions using the 313-nm laser beam (which co-propagates with the $782 \mathrm{~nm}$ laser beam). Repeating such measurements for various values of the trap r.f. voltage allows separating the axial and the radial component (the latter being due to the residual projection of the laser beam onto the radial direction). We find that the axial micromotion component is dominant, which is explained as follows. First, the 782-nm laser is aligned parallel to the trap axis, so that the radial micromotion amplitude is suppressed by the near-zero angle between the 782-nm laser and the radial directions. Second, as the surrounding $\mathrm{Be}^{+}$ions shield the $\mathrm{HD}^{+}$from static radial offset fields, the $\mathrm{HD}^{+}$ions are trapped symmetrically about (and close to) the nodal line of the r.f. field. These two conditions limit the contribution of the radial micromotion to well below the measured axial amplitude of 11(4) $\mathrm{nm}$. The resulting sidebands are included in the spectral line shape function, leading to an additional frequency shift of $-55(20) \mathrm{kHz}$ with respect to the case of zero micromotion.

After correcting for all systematic frequency shifts (Table 1), we finally obtain $v_{0}=383,407,177.38(41) \mathrm{MHz}$.

\section{Discussion}

Our frequency value $v_{0}$ of the $(v, L):(0,2)-(8,3)$ transition is in good agreement with the more accurate theoretical value ${ }^{2}$ $v_{0 \text {,theo }}=383,407,177.150(15) \mathrm{MHz}$. The contribution of the QED terms $1,2,18$ to this frequency amounts to $-1547.225(15) \mathrm{MHz}$ ( $-4035.46(4)$ p.p.b.), and our measurement therefore confirms the validity of QED in a molecular system at an unprecedented level of $2.7 \times 10^{-4}$. We furthermore note that our measurement is sensitive to (and in agreement with) QED terms up to order $m_{\mathrm{e}} \alpha^{7}$, given that the $m_{\mathrm{e}} \alpha^{7}$ term contributes $780(15) \mathrm{kHz}$ (that is, 2.03(4) p.p.b.) to the transition frequency.

Salumbides et al. ${ }^{5}$ showed that spectroscopic tests of molecular QED can be used to set upper bounds on a hypothetical 'fifth force', acting between hadrons at separations of the order of $1 \AA$, and arising from the exchange of unknown, massive virtual particles. Modelling such a hadron-hadron interaction with a

Table 1 | Systematic shifts and uncertainty budget.

\begin{tabular}{lccc} 
Origin & Shift & \multicolumn{1}{c}{ Uncertainty } \\
\cline { 2 - 4 } & (MHz) & (MHz) & (p.p.b) \\
\hline Resolution (statistical fit error) & 0 & 0.33 & 0.85 \\
Doppler effect due to chemistry & $-0.25^{\star}$ & 0.25 & 0.66 \\
Ignoring population L=6 in rate equations & 0 & 0.032 & 0.083 \\
Doppler effect due to micromotion & $-0.055^{\star}$ & 0.020 & 0.052 \\
Frequency measurement & 0 & 0.010 & 0.026 \\
BBR temperature & 0 & 0.005 & 0.013 \\
Zeeman effect & -0.0169 & 0.003 & 0.008 \\
Stark effect & -0.0013 & 0.0001 & 0.0004 \\
Electric-quadrupole shift & 0 & 0.0001 & 0.0003 \\
2nd-Order Doppler effect & $0^{\dagger}$ & 0.000005 & 0.00001 \\
Total & -0.0182 & 0.41 & 1.1
\end{tabular}

BBR, blackbody radiation; p.p.b., parts per billion.

*These frequency shifts are accounted for by the spectral fit function, rather than being subtracted from $v_{0, \text { fit- }}$

$\dagger$ These values are non-zero, but negligibly small and therefore ignored here. 
Yukawa-type potential of the form $\hbar c \alpha_{5} e^{-r / \lambda} / r$ and computing the resulting frequency shift to $v_{0}$, we can exploit the 1 p.p.b. agreement between theory and experiment to rule out (at the 90\% confidence level) interactions at a range $\lambda=1-2 \AA$ (corresponding to force-carrying particles with mass $m_{\mathrm{Y}}=1-2 \mathrm{keV} c^{-2}$ ) with an interaction strength relative to the fine-structure constant, $\left|\alpha_{5} / \alpha\right|$, larger than $5-8 \times 10^{-10}$ (Fig. 4). In a similar way, applying the Arkani-Hamed-Dimopoulos-Dvali formulation to probe the effect of compactified higher dimensions on energy levels in molecules ${ }^{6}$, we place an improved upper bound of $0.5 \mu \mathrm{m}$ on the compactification radius of higher dimensions in 11-dimensional M-theory.

Four decades ago, Wing et al. ${ }^{4}$ suggested that molecular theory could one day be used to translate measured vibrational frequencies of $\mathrm{HD}^{+}$to a new value of the proton-electron mass ratio, $\mu$. The high accuracy of our result and the good agreement with theory, which assumes the 2010 Committee on Data for Science and Technology (CODATA) recommended value $\mu_{\text {CODATA } 10}$, now allow the first determination of $\mu$ from



Figure 4 | Constraint on fifth forces between hadrons at the Ångstrom scale. The high accuracy of our result and the good agreement with theory allow constraining the strength of hypothetical hadron-hadron interactions ${ }^{5}$ with a characteristic range of the order of $1 \AA$, mediated through virtual particles with a mass of order $1 \mathrm{keV}^{-2}$. The present result (solid curve) improves several times on the previous best constraints obtained from $\mathrm{HD}^{+}$(dashed curve) and neutral molecular hydrogen $\left(\mathrm{D}_{2} \text {, dotted curve }\right)^{5}$, ruling out interactions with strengths $\left|\alpha_{5} / \alpha\right|>5 \times 10^{-10}$ ( $90 \%$ confidence level). The yellow region remains unexplored by this experiment. molecular vibrations. Previously ${ }^{19}$ we had derived the sensitivity relation $\delta \mu / \mu=-2.66 \delta v_{0} / v_{0}$, which we employ to adjust $\mu_{\mathrm{CODATA} 10}$ to a new value, $\mu_{\mathrm{HD}+}$, such that the theoretical frequency matches our experimental value. We thus find $\mu_{\mathrm{HD}+}=1,836.1526695(53)$, with a relative uncertainty of 2.9 p.p.b., which approaches that of the values taken into account in the 2010 CODATA adjustment ${ }^{20}$. For example, the value reported by Farnham et al. is only a factor of 1.3 more precise than our result ${ }^{21}$. Although the precision of a very recent determination by Sturm et al. is still 31 times higher ${ }^{22}$, we point out that our method yields $\mu$ as a single parameter from molecular vibrations, whereas most other $\mu$ values are ratios of individual determinations of the electron and proton relative atomic masses (exceptions are $\mu$ determinations from atomic laser spectroscopy $\left.{ }^{23,24}\right)$. Therefore, the agreement of $\mu_{\mathrm{HD}}+$ with all other values of $\mu$ forms an additional consistency check of the various (and vastly different) methods used (Fig. 5b). In particular, it implies that relativistic quantum mechanics and QED consistently describe at the few p.p.b. level such diverse systems as the bound electron ${ }^{22,25-27}$, antiprotonic helium ${ }^{23}$ and the molecular hydrogen ion. Furthermore, of all the methods that produce $\mu$ as a single parameter, our approach is surpassed only by spectroscopy of antiprotonic helium, which is 2.3 times more precise but additionally requires charge, parity and time reversal invariance ${ }^{23}$ (Fig. 5a).

In principle, the transition frequency $v_{0}$ depends on the value of other fundamental constants as well, such as the deuteronproton mass ratio $7, m_{\mathrm{d}} / m_{\mathrm{p}}$, the fine structure constant, and the proton charge radius ${ }^{1,2}$. However, the sensitivity of $v_{0}$ to changes in $\mu$ is known to be three times larger than the second largest one, the $m_{\mathrm{d}} / m_{\mathrm{p}}$ sensitivity ${ }^{7}$. Moreover, if we propagate the uncertainties of the 2010 CODATA values of the fundamental constants through the sensitivity relations, we find that the relative contributions by $\mu, m_{\mathrm{d}} / m_{\mathrm{p}}, \alpha$ and the proton radius to the theoretical uncertainty of $v_{0}$ are 154, 11.6, 0.004 and 5.13 parts per trillion, respectively. We therefore conclude that $\mu$ is the correct parameter to constrain.

The error budget in Table 1 reveals that the experimental uncertainty is limited by Doppler broadening. To overcome this, more involved Doppler-free two-photon spectroscopy of $\mathrm{HD}^{+}$ has been proposed ${ }^{9}$, which should reduce the uncertainty to below $1 \times 10^{-12}$. This should enable a comparison between theory and experiment that would not only test the QED description of chemically bonded particles at an unprecedented

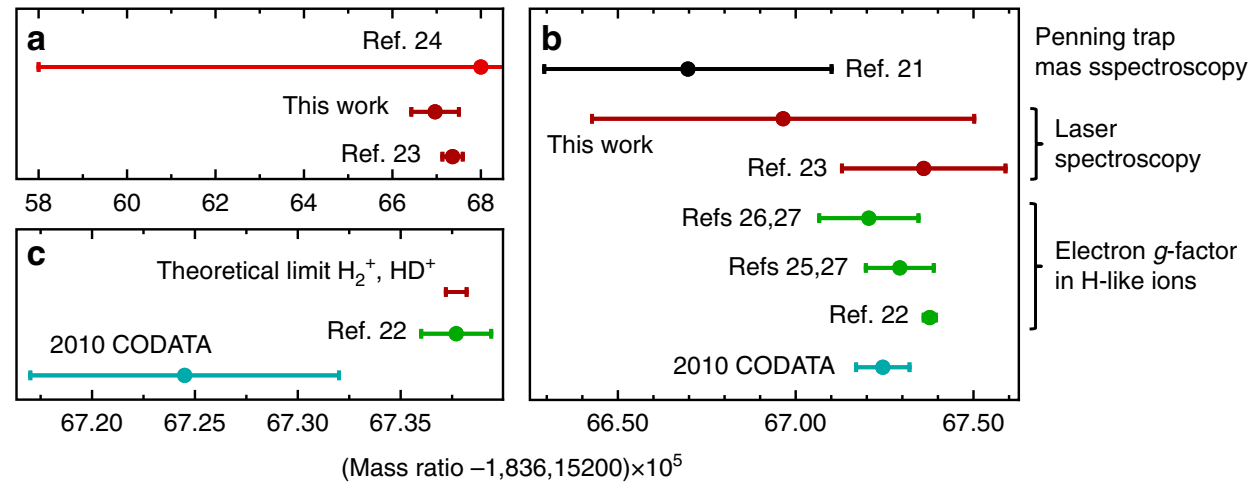

Figure 5 | Determination of the proton-electron mass ratio. (a) Comparison of values of $\mu$ obtained as a single parameter from laser spectroscopy. Shown are results from atomic hydrogen ${ }^{24}$, atomic antiprotonic helium (which assumes charge, parity and time reversal invariance) ${ }^{23}$ and molecular spectroscopy (this work). Error bars represent one standard deviation. (b) Overview of the values included in the 2010 CODATA value of $\mu$, obtained through different methods, in comparison with this work. (c) If experimental accuracy is improved beyond the $\sim 1 \times 10^{-11}$ relative uncertainty of state-ofthe-art QED theory ${ }^{1,2}, \mu$ could in principle be determined from the molecular hydrogen ion with a precision (red bar), which exceeds that of the current best $\mu$ determinations. 
level, but also produce a new value of $\mu$ with an uncertainty below 0.1 p.p.b., surpassing the most precise determination of $\mu$ obtained from independent electron and proton relative atomic mass measurements ${ }^{22}$, which represent a completely different method (Fig. 5c). Our work demonstrates the potential of molecular hydrogen ions for the determination of mass ratios of fundamental particles, as well as stringent tests of QED, and searches for new physics.

\section{Methods}

Experimental procedure. We typically store $40-85 \mathrm{HD}^{+}$ions in a linear r.f. trap, together with $\sim 750 \mathrm{Be}^{+}$ions, which are laser cooled to a temperature of $5-10 \mathrm{mK}$ using a continuous-wave (CW) 313-nm laser beam propagating along the symmetry $(z)$ axis of the trap. As a consequence, only the axial motion of the $\mathrm{Be}^{+}$ions is laser-cooled directly. However, the three-dimensional extent of the Coulomb crystal ensures good coupling of the axial motion to the radial motion of the $\mathrm{Be}^{+}$ and $\mathrm{HD}^{+}$ions, so that both $\mathrm{Be}^{+}$and $\mathrm{HD}^{+}$are efficiently cooled in all three dimensions. Although larger numbers of ions may be trapped, smaller ion numbers ensure better reproducibility of the experimental conditions. The stronger confinement of $\mathrm{HD}^{+}$by the pseudopotential leads to the formation of a string or zig-zag structure of $\mathrm{HD}^{+}$ions along the nodal line of the r.f. field, which coincides with the $z$ axis. $\mathrm{Be}^{+}$ions arrange themselves in a three-dimensional ellipsoidal structure surrounding the $\mathrm{HD}^{+}$. At temperatures below $0.1 \mathrm{~K}$, the two-species ion ensemble solidifies into a Coulomb crystal. $313 \mathrm{~nm}$ fluorescence photons emitted by $\mathrm{Be}^{+}$are imaged onto an electron multiplied charge-coupled device camera and a photomultiplier tube. We obtain a measure of the number of trapped $\mathrm{HD}^{+}$ions by resonantly driving their radial secular motion $(\sim 800 \mathrm{kHz})$ using an a.c. electric field ${ }^{19}$. MD simulations indicate that this 'secular excitation' heats up and melts the ion ensemble, heating the $\mathrm{Be}^{+}$ions to an average temperature (in the $z$-direction) $T_{\mathrm{Be}+\text {, av }}$ proportional to the number of $\mathrm{HD}^{+}, N_{\mathrm{HD}+}$. Typically, $T_{\mathrm{Be}+\text {, av }}=2-4 \mathrm{~K}$. For a fixed cooling-laser detuning $\Delta=-18 \Gamma$ (with $\Gamma=2 \pi \times 19.4 \mathrm{MHz}$, the natural linewidth of the $313 \mathrm{~nm}$ cooling transition), this temperature rise leads to Doppler broadening and, thus, to a significant rise in the 313-nm fluorescence rate with average value $F$. Whereas previous work approximated the fluorescence rise versus $N_{\mathrm{HD}}+$ by a linear dependence ${ }^{28}$, we realistically model the (nonlinear) dependence of $F$ on $N_{\mathrm{HD}+}$, and take this into account in our analysis (see Spectral line shape function for fitting).

To excite the $(v, L):(0,2)-(8,3)$ rovibrational transition at $782 \mathrm{~nm}$, we send a CW 782 -nm laser beam along the $z$ axis, counter-propagating the 313-nm laser beam. The 782-nm radiation is obtained from a titanium:sapphire laser with a linewidth of $0.5 \mathrm{MHz}$, which is frequency locked to an optical frequency comb laser as follows. The frequency of the optical beat note of the 782-nm laser with a nearby mode of the comb is measured by a counter every $30 \mathrm{~ms}$. From the measured beat-note frequency, the comb repetition rate, comb carrier-envelope offset frequency and the comb mode number, we determine the laser's optical frequency. The counter output is fed into a digital feedback loop, which controls the 782-nm laser so as to stabilize the beat-note frequency to a pre-set value. The comb is fully referenced to a global positioning system (GPS) disciplined rubidium atomic clock (providing long-term relative accuracy at the level of $2 \times 10^{-12}$ ), and the frequency uncertainty of our optical frequency measurement system $(10 \mathrm{kHz})$ is limited solely by the frequency instability of the locked $782 \mathrm{~nm}$ laser averaged over the $10 \mathrm{~s}$ of REMPD. To detect excitation to the $v=8$ state by the $782 \mathrm{~nm}$ laser, we overlap this beam with a CW 532-nm laser beam, leading to REMPD of HD ${ }^{+}$ (Fig. 1a). An experimental cycle looks as follows. We first load a fresh sample of $\mathrm{HD}^{+}$and find a measure, $F_{\mathrm{i}}$, of the initial ion number, $N_{\mathrm{i}}$, by secular excitation. We subsequently lower the 313-nm cooling laser intensity and detuning to reduce the ion temperature to $\sim 10 \mathrm{mK}$, and expose the ions to the 313,782 and $532 \mathrm{~nm}$ lasers for $10 \mathrm{~s}$. Afterwards, we apply secular excitation to determine the fluorescence level $F_{\mathrm{f}}$ corresponding to the remaining ion number, $N_{f}$, and we define a signal $S=\left(F_{\mathrm{i}}-F_{\mathrm{f}}\right) / F_{\mathrm{i}}$. Repeating this cycle for different values of the 782-nm laser frequency, $v$, we obtain a spectrum consisting of 1,772 points (Fig. 2a). Noise in the spectrum is primarily due to the stochastic nature of REMPD of small $\mathrm{HD}^{+}$ ensembles with a mean rotational-hyperfine occupancy of the order of one ion per state.

Spectral line shape function for fitting. Hyperfine rate equations allow computing the evolution of a sample of $\mathrm{HD}^{+}$ions, with an initial thermal rotational distribution ${ }^{29}$ corresponding to $T=300(5) \mathrm{K}$, under the influence of REMPD lasers, BBR and losses due to chemical reactions with background-gas molecules (Fig. 1b,d). Population in rotational states with $L=0-5$ is included, thus ignoring the $2.4 \%$ population in $L=6$ and higher. Accurate hyperfine line strengths at the magnetic sub-state level are obtained by extending the approach of Koelemeij ${ }^{30}$ so as to include hyperfine structure and the Zeeman effect. The response to the 782-nm laser of each hyperfine level is modelled using a Doppler-broadened profile based on $q$-Gaussians. For the assessment of the Zeeman effect, the hyperfine rate equations are adapted to include Zeeman line shifts and broadening due to hyperfine line splitting (which remains much smaller than the $16-\mathrm{MHz}$ Doppler width). The $q$ parameter is determined from realistic
MD simulations (which take into account the time-dependent trap potential and momentum changes by scattering of photons from the cooling laser) with an uncertainty limited by the minimum and maximum expected rates of heating events. Solving the rate equations allows predicting the fractional loss of $\mathrm{HD}^{+}, \gamma$, as a function of the 782-nm laser frequency relative to the hyperfine-less frequency, $v-v_{0}$, the intensity, $I_{\mathrm{L}}$, of the 782 -nm laser and the $\mathrm{HD}^{+}$motional temperature, $T_{\mathrm{HD}+}$. We obtain a smooth, continuous spectral line shape function for fitting as follows. First, we compute $\gamma$ on a dense three-dimensional grid of values $\left(v, I_{\mathrm{L}}\right.$, $\left.T_{\mathrm{HD}+}\right)$, which we subsequently interpolate to obtain a four-dimensional function $\gamma\left(v-v_{0, \text { fit }}, I_{\mathrm{L}}, T_{\mathrm{HD}+}\right)$. Using the shorthand notation $\gamma\left(v-v_{0, \text { fit }}, I_{\mathrm{L}}, T_{\mathrm{HD}+}\right)=\gamma$, and making use of the linear dependence of $T_{\mathrm{Be}+\text {,av }}$ on $N_{\mathrm{HD}}$ found from $\mathrm{MD}$ simulations, the average $\mathrm{Be}^{+}$temperatures during secular excitation of $\mathrm{HD}^{+}$ before and after REMPD are given by $T_{\mathrm{i}} \equiv T_{\mathrm{Be}+\text {, av }}\left(N_{\mathrm{i}}\right)$ and $T_{\mathrm{f}} \equiv T_{\mathrm{Be}+\text {, av }}\left(\gamma N_{\mathrm{i}}\right)$, respectively. We consecutively insert these temperature values in the scattering rate function $g$, defined as

$$
g\left(T_{\mathrm{Be}^{+}}\right)=\frac{\Gamma}{2} \sqrt{\frac{m_{\mathrm{Be}^{+}}}{2 \pi k_{\mathrm{B}} T_{\mathrm{Be}^{+}}}} \int_{-\infty}^{\infty} \frac{s}{1+s+4\left(\Delta-k v_{k}\right)^{2} / \Gamma^{2}} \exp \left(-\frac{m v_{k}^{2}}{2 k_{\mathrm{B}} T_{\mathrm{Be}^{+}}}\right) d v_{k},
$$

which includes the beryllium mass, $m_{\mathrm{Be}+}$, and the fixed $313 \mathrm{~nm}$ laser detuning, wave vector, $\mathbf{k}$, and saturation parameter, $s$. The integration is performed over the distribution of $\mathrm{Be}^{+}$velocities $\mathbf{v}$ along $\mathbf{k}$, that is, $v_{k} \equiv \mathbf{k} \cdot \mathbf{v} /|\mathbf{k}|$. This function is used to model the fluorescence rise $F$ observed in the experiment, and allows us to construct a five-dimensional fit function $G\left(v-v_{0, \text { fit }}, I_{\mathrm{L}}, T_{\mathrm{HD}+}, T_{\mathrm{i}}\right) \equiv\left[g\left(T_{\mathrm{i}}\right)-g\left(T_{\mathrm{f}}\right)\right] /$ $\left[g\left(T_{\mathrm{i}}\right)-g_{0}\right]$, with $g_{0}$ the steady-state scattering rate before secular excitation. The function $G$ realistically models the signal $S$ (Fig. 2c), and is used for fitting.

We employ the above model also to estimate the uncertainties due to several systematic effects. For example, MD simulations predict a slight increase of $T_{\mathrm{HD}}+$ and $q$ with increased REMPD rates in the scenario of inefficient thermalization of fast $\mathrm{D}^{+}$. We can estimate the frequency shift because of this effect by making both $T_{\mathrm{HD}}+$ and $q$ REMPD-rate dependent. This leads to an additional frequency uncertainty of $61 \mathrm{kHz}$, which is included in the error associated with Doppler effects due to chemistry (Table 1). From our model, we furthermore deduce that ignoring states with $L \geq 6$ introduces an uncertainty of $32 \mathrm{kHz}$, whereas the possible $5 \mathrm{~K}$ error in the BBR temperature estimate (which takes into account day-to-day temperature variations and the possibility that the trap electrodes may be at a slightly higher temperature due to r.f. dissipation) translates to a $5-\mathrm{kHz}$ effect.

\section{References}

1. Korobov, V. I., Hilico, L. \& Karr, J.-Ph. $m \alpha^{7}$-Order corrections in the hydrogen molecular ions and antiprotonic helium. Phys. Rev. Lett. 112, 103003 (2014).

2. Korobov, V. I., Hilico, L. \& Karr, J.-Ph. Theoretical transition frequencies beyond 0.1 p.p.b. accuracy in $\mathrm{H}_{2}^{+}, \mathrm{HD}^{+}$, and antiprotonic helium. Phys. Rev. A 89, 032511 (2014).

3. Bressel, U. et al. Manipulation of individual hyperfine states in cold trapped molecular ions and application to $\mathrm{HD}^{+}$frequency metrology. Phys. Rev. Lett. 108, 183003 (2012).

4. Wing, W. H., Ruff, G. A., Lamb, Jr. W. E. \& Spezeski, J. J. Observation of the infrared spectrum of the hydrogen molecular ion $\mathrm{HD}^{+}$. Phys. Rev. Lett. 36, 1488-1491 (1976).

5. Salumbides, E. J. et al. Bounds on fifth forces from precision measurements on molecules. Phys. Rev. D 87, 112008 (2013).

6. Salumbides, E. J., Schellekens, A. N., Gato-Rivera, B. \& Ubachs, W. Constraints on extra dimensions from precision molecular spectroscopy. N. J. Phys. 17, 033015 (2015)

7. Schiller, S. \& Korobov, V. Tests of time independence of the electron and nuclear masses with ultracold molecules. Phys. Rev. A 71, 032505 (2005).

8. Schiller, S., Bakalov, D. \& Korobov, V. I. Simplest molecules as candidates for precise optical clocks. Phys. Rev. Lett. 113, 023004 (2014).

9. Tran, V. Q., Karr, J.-Ph., Douillet, A., Koelemeij, J. C. J. \& Hilico, L. Two-photon spectroscopy of trapped $\mathrm{HD}^{+}$ions in the Lamb-Dicke regime. Phys. Rev. A 88, 033421 (2013).

10. Karr, J.-Ph. $\mathrm{H}_{2}^{+}$and $\mathrm{HD}^{+}$: candidates for a molecular clock. J. Mol. Spectrosc. 300, 37-43 (2014)

11. Koelemeij, J. C. J., Roth, B., Wicht, A., Ernsting, I. \& Schiller, S. Vibrational spectroscopy of $\mathrm{HD}^{+}$with 2-p.p.b. accuracy. Phys. Rev. Lett. 98, 173002 (2007).

12. Bakalov, D., Korobov, V. I. \& Schiller, S. High-precision calculation of the hyperfine structure of the $\mathrm{HD}^{+}$ion. Phys. Rev. Lett. 97, 243001 (2006).

13. Berkeland, D. J., Miller, J. D., Bergquist, J. C., Itano, W. M. \& Wineland, D. J. Minimization of ion micromotion in a Paul trap. J. Appl. Phys. 83, 5025-5033 (1998).

14. Bakalov, D. \& Schiller, S. The electric quadrupole moment of molecular hydrogen ions and their potential for a molecular ion clock. Appl. Phys. B 114, 213-230 (2014).

15. Roth, B., Koelemeij, J. C. J., Daerr, H. \& Schiller, S. Rovibrational spectroscopy of trapped molecular hydrogen ions at millikelvin temperatures. Phys. Rev. A 74, 040501(R) (2006) 
16. Wineland, D. J. et al. Experimental issues in coherent quantum-state manipulation of trapped atomic ions. J. Res. Natl Inst. Stand. Technol. 103, 259 (1998).

17. Umarov, S., Tsallis, C. \& Steinberg, S. On a $q$-central limit theorem consistent with nonextensive statistical mechanics. Milan J. Math 76, 307-328 (2008).

18. Korobov, V. I. Relativistic corrections of $m \alpha^{6}$ order to the rovibrational spectrum of $\mathrm{H}_{2}^{+}$and $\mathrm{HD}^{+}$molecular ions. Phys. Rev. A 77, 022509 (2008).

19. Koelemeij, J. C. J., Noom, D. W. E., De Jong, D., Haddad, M. A. \& Ubachs, W. Observation of the $v^{\prime}=8-v=0$ vibrational overtone in cold trapped $\mathrm{HD}^{+}$. Appl. Phys. B 107, 1075-1085 (2012).

20. Mohr, P. J., Taylor, B. N. \& Newell, D. B. CODATA recommended values of the fundamental physical constants: 2010. Rev. Mod. Phys. 84, 1527-1605 (2012).

21. Farnham, D. L., Van Dyck, Jr. R. S. \& Schwinberg, P. B. Determination of the electron's atomic mass and the proton/electron mass ratio via Penning trap mass spectroscopy. Phys. Rev. Lett. 75, 3598-3601 (1995).

22. Sturm, S. et al. High-precision measurement of the atomic mass of the electron. Nature 506, 467-470 (2014).

23. Hori, M. et al. Two-photon laser spectroscopy of antiprotonic helium and the antiproton-to-electron mass ratio. Nature 475, 484-488 (2011).

24. De Beauvoir, B. et al. Absolute frequency measurement of the 2S-8S/D transitions in hydrogen and deuterium: new determination of the Rydberg constant. Phys. Rev. Lett. 78, 440-443 (1997).

25. Beier, T. et al. New determination of the electron's mass. Phys. Rev. Lett. 88 , 011603 (2002)

26. Verdu, J. et al. Electronic g factor of hydrogenlike oxygen ${ }^{16} \mathrm{O}^{7+}$. Phys. Rev. Lett. 92, 093002 (2004).

27. Mohr, P. J., Taylor, B. N. \& Newell, D. B. CODATA recommended values of the fundamental physical constants: 2006. Rev. Mod. Phys 80, 633-730 (2008).

28. Schneider, T., Roth, B., Duncker, H., Ernsting, I. \& Schiller, S. All-optical preparation of molecular ions in the rovibrational ground state. Nat. Phys. 6, 275-278 (2010).

29. Koelemeij, J. C. J., Roth, B. \& Schiller, S. Blackbody thermometry with cold molecular ions and application to ion-based frequency standards. Phys. Rev. A 76, 023413 (2007).

30. Koelemeij, J. C. J. Infrared dynamic polarizability of $\mathrm{HD}^{+}$rovibrational states. Phys. Chem. Chem. Phys. 13, 18844 (2011).

\section{Acknowledgements}

We are indebted to J. Bouma, T. Pinkert and R. Kortekaas for technical assistance, and to V. Korobov, E. Hudson and R. Gerritsma for fruitful discussions. We thank E. Salumbides for providing the curve for $\mathrm{D}_{2}$ in Fig. 4. This research was funded through the Netherlands Foundation for Fundamental Research on Matter (FOM), the COST action MP1001 IOTA and the Dutch-French bilateral Van Gogh Programme. SURFsara (http://www.surfsara.nl) is acknowledged for the support in using the Lisa Compute Cluster for MD simulations. J.C.J.K. thanks the Netherlands Organisation for Scientific Research (NWO) and the Netherlands Technology Foundation (STW) for support.

\section{Author contributions}

J.B. and J.C.J.K built the apparatus, and acquired and analysed data. K.S.E.E. provided the optical frequency measurement system. J.-Ph.K., L.H., J.B. and J.C.J.K. evaluated systematic frequency shifts and performed simulations. W.U. and J.C.J.K. conceived and supervised the experiment. J.B. and J.C.J.K. wrote the manuscript, which was edited and approved by all co-authors.

\section{Additional information}

Competing financial interests: The authors declare no competing financial interests.

Reprints and permission information is available online at http://npg.nature.com/ reprintsandpermissions/

How to cite this article: Biesheuvel, J. et al. Probing QED and fundamental constants through laser spectroscopy of vibrational transitions in $\mathrm{HD}^{+}$. Nat. Commun. 7:10385 doi: $10.1038 /$ ncomms10385 (2016).

(c) (i) This work is licensed under a Creative Commons Attribution 4.0 International License. The images or other third party material in this article are included in the article's Creative Commons license, unless indicated otherwise in the credit line; if the material is not included under the Creative Commons license, users will need to obtain permission from the license holder to reproduce the material. To view a copy of this license, visit http://creativecommons.org/licenses/by/4.0/ 